\title{
The Structure Optimization of T-slots Dry Gas Seal Faces
}

\author{
Xuebin Li, Kun Wang and Biao Du \\ School of mechanical Engineering, Anhui University of Science and Technology, Huainan Anhui China 232001
}

\begin{abstract}
Numerical simulation method based on CFD simulation soft, Launched a T-slots dry gas seal structure optimization. In the case of only changing the Circumferential ratio of T-slot structure of initial improvements, further optimized on the basis of the initial improvements, conclusion that lug boss groove seal faces. Similarly to the boss groove seals flow field simulation, the gas film pressure distribution of the seal face of the boss groove is simulated and pressure distribution in the boss groove. The obtained results are compared with the simulation results of T-slot. Results show that full use of the slot space, effectively reducing the dry gas seal face wear, help to improve the working life of dry gas seal and sealing effect, when the sealing end face is a boss groove and the circumferential ratio $=0.5$.
\end{abstract}

Keywords-T-slots; dry gas seal; CFD simulation; structure optimization

\section{INTRODUCTION}

At present optimization method for $\mathrm{T}$-slot structure is generally: under the given working conditions, one or two of the structure parameters, such as groove depth, slot number, channel width ratio, slot bar ratio and helix angle, are used as variables, fixed other parameters to obtain the variation law of the sealing characteristic parameter with the variable structure parameter. A single optimum sealing characteristics parameters as the optimization target obtain the structural parameters of the preferred range. This method takes into account only the structural parameters alone, ignoring the interaction between structural variables of the objective function, and thus the resultant T-slot structure parameter optimization results have greater limitations, and the preferred structure of the T-slot given parameter values more dispersed, so it is still not yet formed a clear T-slot design theory and methods.

This paper is a study of speed in the $n=9000 \mathrm{r} / \mathrm{min}$ case, circumferential ratio of dry gas seal faces with the relationship between the parameters. The regularity of the change of the composite curve is close to the maximum value of the opening force of the end face and the stiffness of the film when compared to 0.5 , at the same time to change the structure of the T-slot optimization. On this basis, provide a reference for engineering design and selection.

\section{T-SOLt DRY GAS SEAL FlOW FIELD SIMULATION}

\section{A. T-slot Three Dimensional Solid Model}

Above parameters are commonly used in the parameters of the T-slot, on the basis of the commonly used $\mathrm{T}$ groove face parameters, in this paper, Solid Works is used to model the end face of T-slot. The model is shown in Figure I.
Considering the particularity of Fluent flow analysis, the three-dimensional modeling of Fluent used in the model, and in the thickness direction 1000 times magnification, as shown in Figure II.

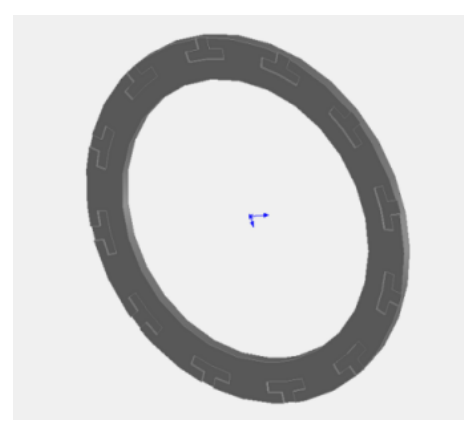

FIGURE I. USING SOLIDWORKS BUILT T-GROOVE CELL FACE
MODEL

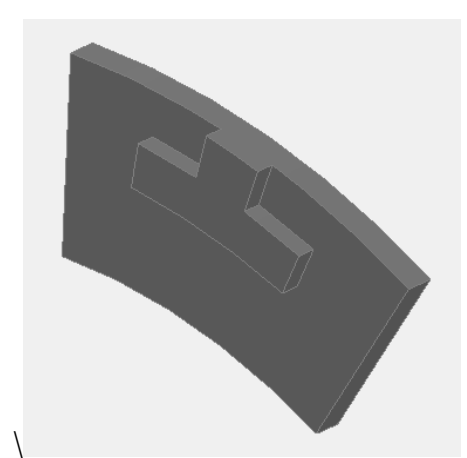

FIGURE II. MAGNIFIED 1000 TIMES TIMES THE MODEL

\section{B. Fluent Solution}

The model of the solver is selected, and the flow field of the end face of the calculation model should belong to laminar flow, in the calculation model, thelaminar flow is selected. Then define the basic physical properties of the fluid, the subject of analysis of the use of the seal gas for the air. Fluent built a variety of physical properties of the air. Boundary conditions set by reference ${ }^{[2]}$ shows that the outer diameter of the agency for the import of the inlet pressure $\mathrm{P}_{0}=4.5852 \mathrm{MPa}$, the inside diameter is air, outlet pressure $P_{i}=0.013 \mathrm{MPa}$, air dynamic viscosity $\mu=1.8 \times 10^{-5}$ pas, set up a discrete format with relaxation factor with relaxation factor, setting the solution process monitoring parameters, intializes.

After the iteration is completed, the pressure distribution of the T-slot dry gas seal at the $\mathrm{z}=0$ is obtained when the gas film thickness is $3 \mathrm{um}$. 


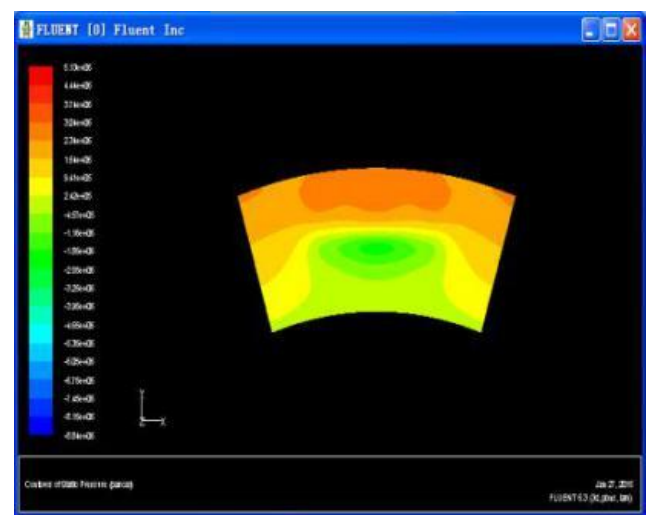

FIGURE III. PRESSURE DISTRIBUTION ON THE CELL SURFACE MEMBRANE

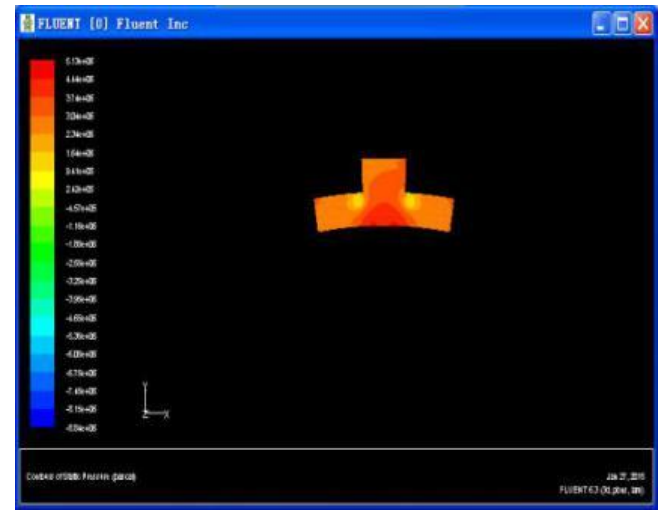

FIGURE IV. T-SLOT FILM PRESSURE DISTRIBUTION

\section{RESUltS ANALYSIS AND DISCUSSION}

A. Relationship between the Parameters of the Gas Film of the End Face and the Circumferential Ratio Al

In the evaluation of the merits of a seal, only when the computer simulation is not enough, but also to compare the sealing parameters, the size of the seal parameters is decided to dry gas seal is good or bad standard. The performance parameters of the dry gas seal end face gas film mainly include the opening force $\mathrm{F}_{\text {open }}$, the leakage rate $\mathrm{Q}$, the stiffness of the gas film Kz and the ratio of stiffness to leakage $\Gamma$ and so on.

Listing the circumferential ratio $a_{1}$ at different speed, opening force, leakage volume, gas film stiffness and stiffness to leakage ratio between the curve, as shown in Figure V (a), (b), (c), (d) below:

In this paper, the relationship between the circumferential ratio of dry gas seal face and the parameters is studied in the case of rotational speed $n=9000 \mathrm{r} / \mathrm{min}$. Comprehensive curve pattern, face opening force and film stiffness values close to the maximum when the circumferential ratio ${ }^{a_{1}}=0.5$. Leakage

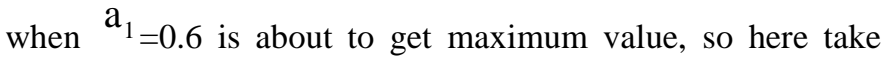
$\mathrm{a}_{1}=0.5$ to existing T-slots for structural optimization.

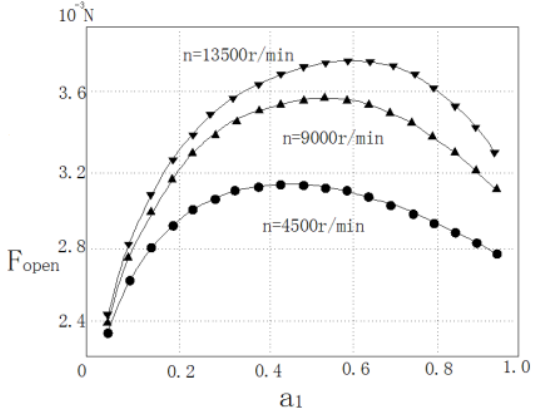

(a) the relationship between the opening force and the circumferential ratio

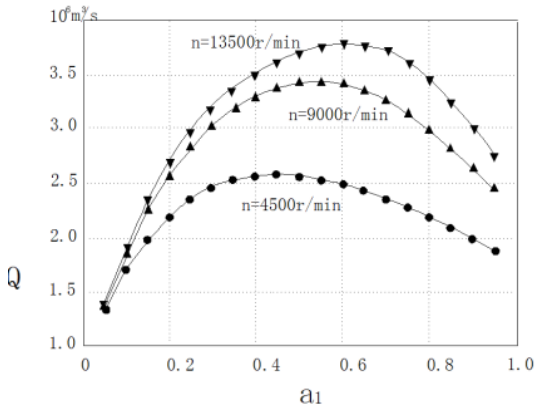

(b) the relationship between the amount of leakage and the circumferential ratio

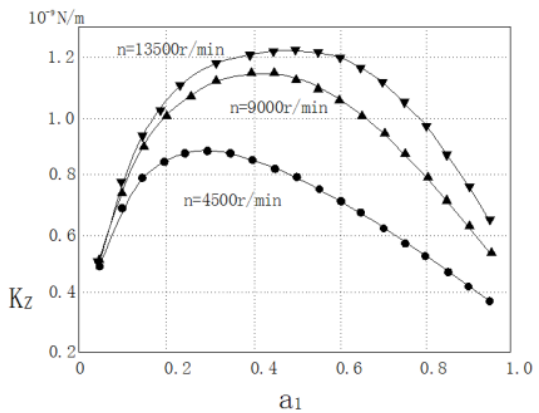

(c) the relationship between the gas film stiffness and the circumferential ratio

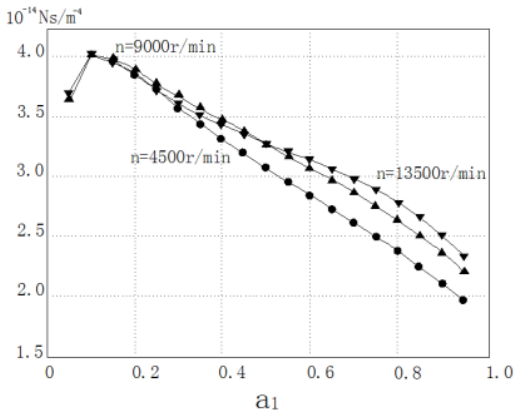

(d) relationship between ratio of stiffness to leakage and circumference ratio

FIGURE V. THE RELATIONSHIP BETWEEN THE PARAMETERS OF THE END FACE AND THE CIRCUMFERENTIAL RATIO

\section{B. Unit Model and Flow Field Simulation After Structural Improvements}

In the case of each of these parameters do not change, change the reference values of $\mathrm{a}_{1}=\mathrm{w}_{1} / \mathrm{w}_{2}$ modeling. In 
the new geometric model, the parameter values of $a_{1}$ are 0.5 , and the geometric modeling is carried out in the Solid Works according to the other parameters mentioned above. In the Gambit software, the calculation model unit is divided into grid and boundary conditions are specified. Then import the Fluent, to set the parameters on cell model and iterative, monitor the residual curve, curve is obtained convergence at the end of the iteration. After the end of the iteration, the pressure distribution of the gas film at the end of $z=0$ in the Fluent can be obtained when the gas film thickness is $3 \mathrm{um}$, and the gas film pressure distribution in the slot is shown in Figure VII.

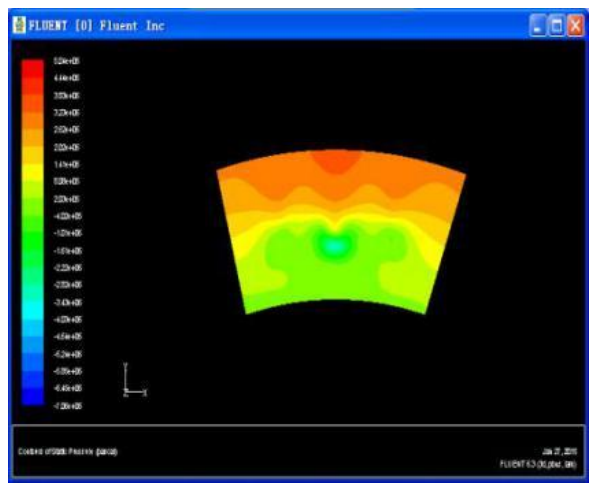

FIGURE VI. GAS FILM PRESSURE DISTRIBUTION ON THE END FACE OF THE CALCULATION UNIT

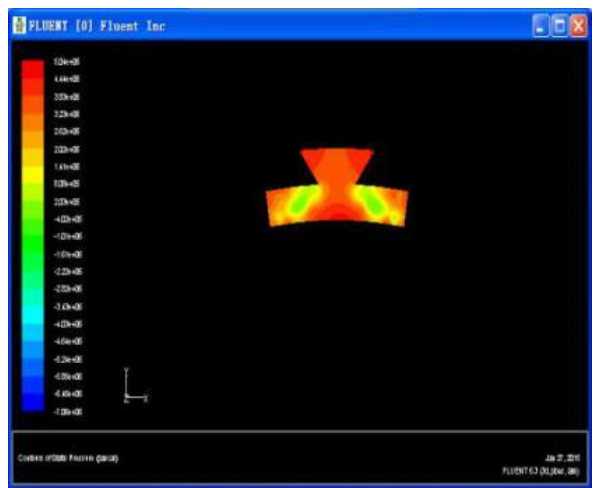

FIGURE VII. TANK GAS PRESSURE DISTRIBUTION AFTER INITIAL IMPROVEMENTS

After the preliminary improvement, the flow field of the end face is simulated by Fluent, and the pressure distribution of the seal face is obtained. After comparing with the original model, the following conclusions are obtained:

(1)The maximum pressure of the T-slot is concentrated in the triangular area of the groove. The maximum pressure is concentrated in the entrance and opposite the entrance at the bottom.

(2)According to the pressure distribution chart analysis, the pressure distribution in the original $\mathrm{T}$ - slot and the model trough is not all, T-slot is not fully utilized, groove part of the gas film pressure is too large, easy to cause the opening force to focus on the area of the gas film pressure.

According to the above analysis, we can know that the model has a large defect, so the structure of the T-slot is optimized further. On the basis of the preliminary improved model, the structure of the middle radius and the inlet of the groove is improved, and the uneven distribution of the gas film pressure is reduced. Specific model improvements are as follows: in the other parameters unchanged, T-slot is changed into a boss form, make $\mathrm{T}$-slot inlet and the middle radius of intersection of rectangular structure is changed into the hypotenuse form with a certain slope. Improved model is shown in Figure VIII. Geometric modeling of the calculation unit used in Fluent, as shown in Figure IX.

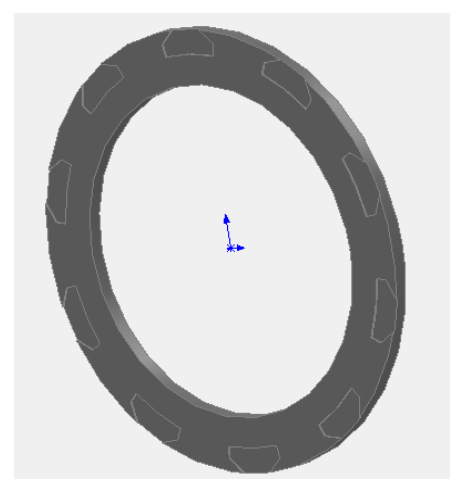

FIGURE VIII. BOSS GROOVE FACE MODEL

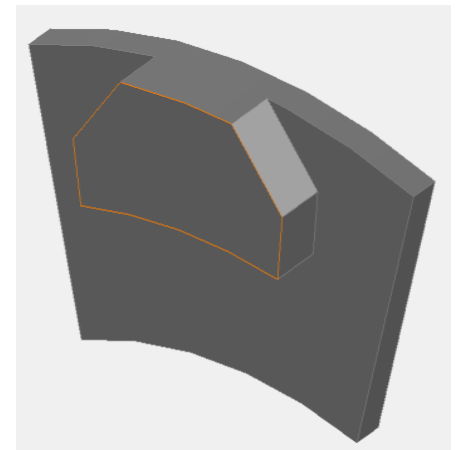

FIGURE IX. CALCULATION UNIT MODEL

Finally, the iterative end of the T-slot is improved, and the results are convergent and the next step is analyzed and calculated. The improved T-slot (boss slot) is in agreement with the above, and the end face of the flow field is simulated, and the simulation results are shown in Figure $\mathrm{X}$ and Figure XI.

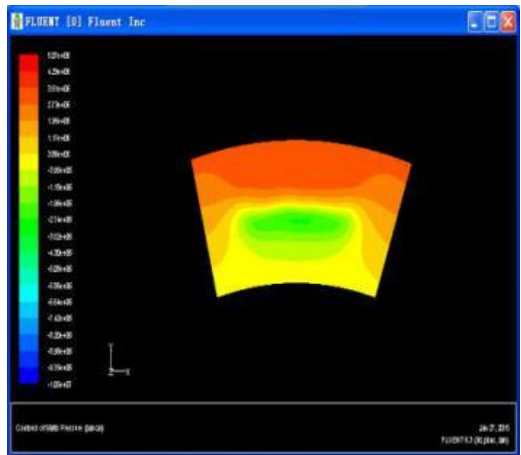

FIGURE X. COMPUTING UNIT FOR GAS FILM FACE PRESSURE DISTRIBUTION 


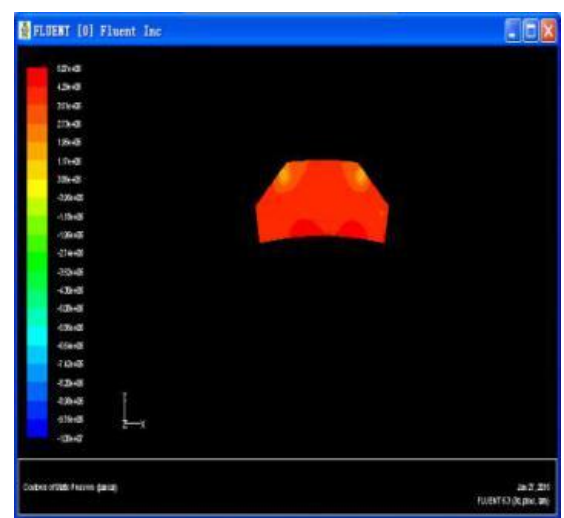

FIGURE XI. FILM PRESSURE DISTRIBUTION IN BOSS TANKS

\section{Analysis and Comparison of the Simulation Results of} Boss Tank and T-slot

By using Fluent software of T-slot sealing surface and improved (lug groove seal flow simulation, two kinds of seal end face of the gas film pressure distribution in the channel and the gas film pressure distribution, through the analysis of the simulation results comparison shows that:

(1)By the comparison of Figure IV and Figure XI, the dynamic pressure of the seal face of the boss tank is larger than that of the $\mathrm{T}$ slot, is that in the dry gas seal end geometric parameters, physical parameters, speed the same. Boss tank seal formed gas film opening is much larger than that of the $\mathrm{T}$ slot.

(2)According to Figure III, the gas film pressure distribution in the $\mathrm{T}$ slot is not uniform. Gas film pressure distribution is on the entrance of axisymmetric, showed a trend of decrease, in the T-shaped slot inlet and the intermediate radius interchange to form stress concentration problems, lug boss groove can average air film pressure and the groove of the gas film pressure distribution is basically the same, make more uniform surface air film pressure distribution and the effect of higher pressure.

(3)As can be seen from Figure IV and Figure XI comparison, T type groove pressure is not uniform, some in the low voltage state, only $\mathrm{T}$ type groove at the entrance and the entrance to the relative triangle (see Figure III) pressure is bigger, so the end surface of the opening force produced did not make full use of the area of the T-shaped groove. In the convex slot, the gas film pressure is relatively large and the distribution of the basic phase is uniform, so the opening force can fully utilize the entire area of the groove surface.

\section{CONCLUSION}

(1)The relationship between the circumferential ratio and the parameters, Select $\mathrm{a}_{1}=0.5$ in the case of other conditions do not change, the t-slot has been improved. And the preliminary improvement of the model modeling, simulation analysis, the pressure distribution of the seal face and groove. Compared with the results of the $\mathrm{T}$ slot, the end face film pressure distribution and the gas film pressure distribution in the channel are obtained, and then the model is optimized.

(2)On the basis of the preliminary improved model, further optimization is made to obtain the seal end face of the boss tank, and the model and flow field analysis are also carried out. The simulation results are compared with the simulation results of the seal face of the T slot, and the conclusion is drawn. From the point of view of simulation, improved method is effectively improves the dry gas seal opening force, improve the groove of the gas film pressure distribution, fully using the space inside the groove and effectively reduce the dry gas seal face wear, help to improve the working life of the dry gas seal and sealing effect.

\section{REFERENCE}

[1] Taozi Li, Qiuxiang Zhang, Jining Cai, Shuangxi Li. Finite element analysis of the steady state characteristics of dry gas seal in T slot, J.Journal of Beijing University of Chemical Technology, 2003,2 (30): 62-58

[2] Yan Wang, Jianjun Sun, Kai Tao, Chenbo Ma, Qiao Tu. Numerical analysis of dry gas seal for T type slot and optimization of groove type, J. friction journal, 2014,34 (4): 420-427

[3] Yuanwei Lu,Wei He,Yuting wu,Weining Ji,Chongfang Ma,Hang Guo.Performance study on compressed air refrigeration system based on single screw expander, J.Energy,2013:762-768

[4] Yufeng Chen, single screw compressor development and outlook, J. air conditioning and refrigeration, 2010,10 (4): 1-4

[5] Qian Zha, Qian Deng, Shi Liang Zha. Major advances in single screw compressor technology, J. compressor technology, 2003 (4): 30-28

[6] Qian Zha, Qian Deng,Shi Liang Zha. The new progress of single screw compressor, J. compressor technology, 2002 (2): 32-35

[7] Chunyan Fan. Discussion on the development status and application of the screw compressor, J. chemical engineering and equipment, 2010 (9): 186-187 